\title{
CLASSIFICAÇÃO HISTOPATOLÓGICA E CORRELAÇÃO CLÍNICA DE 50 CASOS DE HANSENÍASE DIAGNOSTICADOS EM UM HOSPITAL-ESCOLA, SÃO JOSÉ DO RIO PRETO, SP*
}

\author{
HISTOPATHOLOGICAL CLASSIFICATION AND CLINICAL CORRELATION OF 50 LEPROSY CASES \\ FROM A TEACHING HOSPITAL, SÃO JOSÉ DO RIO PRETO, SÃO PAULO STATE, BRAZIL
}

Rafael A. B. Pavani', Emanuele R. Tonolli' ${ }^{1}$, Solange C. G. P. D’Avila²

\begin{abstract}
${ }^{1}$ Acadêmicos do Curso de Medicina, ${ }^{2}$ Docente, Departamento de Patologia e Medicina Legal. Faculdade de Medicina de São José do Rio Preto - FAMERP

CorResPondênCIA: Emanuele R. Tonolli. Rua Piratininga nº 100, Jardim Paulista. São José do Rio Preto - SP, CEP $15060-210$.

E-mail: emanuele_tonolli@hotmail.com
\end{abstract}

Pavani RAB, Tonolli ER, D’Avila SCGP. Classificação histopatológica e correlação clínica de 50 casos de Hanseníase diagnosticados em um Hospital-Escola, São José do Rio Preto, SP. Medicina (Ribeirão Preto) 2008; 41 (2): 188-95.

RESUMO: A classificação mais utilizada para a Hanseníase na prática clínica é a do Congresso de Madrid (1953), com as formas indeterminada, tuberculóide, dimorfa e lepromatosa. Em 1966, Ridley \& Jopling propuseram uma classificação baseando-se no espectro imunológico da doença. Modelo do estudo: Estudo de prevalências. Objetivo: Classificar biópsias de Hanseníase segundo os critérios de Ridley \& Jopling e realizar a correlação anátomo-clínica. Metodologia: Foram revistos 50 pedidos de biópsia e analisadas as lâminas coradas pelos métodos H\&E e Ziehl-Neelsen, utilizando os critérios de Ridley \& Jopling. Resultados: As formas classificadas foram: 9 indeterminadas, 10 tuberculóides, 5 dimorfas tuberculóides, 5 dimorfas lepromatosas, 5 subpolares lepromatosas e 16 lepromatosas. Dos 50 pacientes, 30 eram do sexo masculino, e as formas multibacilares predominaram nesse sexo. Em 2 não se fez hipótese diagnóstica de Hanseníase, e em 30 foi especificada uma ou mais formas, com freqüente discordância entre o diagnóstico histopatológico e a hipótese clínica. Conclusões: A classificação da Hanseníase depende de atenção aos critérios histopatológicos e da correlação com os dados clínicos e imunológicos do paciente.

Descritores: Hanseníase. Patologia. Classificação. Diagnóstico Clínico. Histopatologia.

\section{1- INTRODUÇÃO}

O Brasil é um país endêmico para a Hanseníase, com predomínio de formas multibacilares ${ }^{1,2,3}$. A faixa etária de 15 a 50 anos apresenta prevalência de 60 a $96 \%$ dos casos ${ }^{2,3,4}$, e no sexo masculino ela é superior àquela observada no sexo feminino ${ }^{2,5}$.
As manifestações clínicas da Hanseníase decorrem de complexa resposta imunológica do hospedeiro à infecção, dependendo do tipo de ativação linfocitária, que leva a um espectro imunológico, clínico e histológico característico ${ }^{2,5,6,7}$. Nos pólos deste espectro estão as formas tuberculóide (TT) e lepromatosa (virchowiana, LLp), respectivamente a de 
maior e a de menor resistência à infecção pelo Mycobacterium leprae. A classificação mais utilizada na prática clínica para as formas intermediárias é a de Madrid (1953) ${ }^{8}$, que considera as formas indeterminada e dimorfa (borderline) ${ }^{1,2,5}$. Para fins de tratamento, separam-se as formas indeterminada e tuberculóide como paucibacilares e a dimorfa e lepromatosa como multibacilares.

Em 1966, Ridley \& Jopling propuseram outra classificação baseando-se nas diferenças no grupo de Hanseníase dimorfa segundo a tendência para um dos pólos, e na resposta ao tratamento apresentada por essas subdivisões ${ }^{9}$. Essa classificação visa especialmente os pesquisadores e inclui as formas dimorfa tuberculóide (DT), dimorfa dimorfa (DD) e dimorfa lepromatosa (DL), mantendo as polares, num total de 5 grupos no espectro imunológico, além da forma indeterminada (I). Como observado por Ridley \& Jopling, a forma DD (ou $\mathrm{BB}$, de borderline borderline) é instável, de difícil diferenciação das formas DT e DL, comportando-se geralmente no tratamento como esta última ${ }^{7,9}$. Além disso, eles e outros autores também notaram uma forma diferençável entre a DL e a LLp, posteriormente sendo considerada a forma Subpolar Lepromatosa (LLs), mantendo-se assim na classificação as formas TT, DT, DL, LLs, LLp e I ${ }^{6,10}$. Muitos autores utilizam a classificação original de Ridley \& Jopling ${ }^{9}$, mantendo a forma DD e utilizando ou não a forma LLs ${ }^{2,5,6,11}$.

Para classificar de maneira completa a Hanseníase são necessários dados provenientes da clínica, histopatologia, baciloscopia e teste da lepromina (Mitsuda) $1,2,9,10,12$. A forma apresentada pelo paciente permite a previsão do surgimento de reações imunes e da resposta ao tratamento, além de orientar a instituição do tratamento adequado, diminuindo assim a instalação de seqüelas, recidivas e a transmissão da doença $1,2,9,13,14,15$.

Apesar do arsenal diagnóstico disponível e da efetividade do tratamento, estudos evidenciam dificuldade para se estabelecer o diagnóstico da Hanseníase e sua classificação, com discordância entre o diagnóstico clínico e o achado histopatológico, e mesmo histopatologistas experientes podem ter dificuldade na classificação de Ridley \& Jopling ${ }^{11,16,17}$. Khan et al. (2004) relataram concordância de $66 \%$ entre os achados do exame histopatológico de fragmentos de pele e o diagnóstico clínico de hanseníase ${ }^{17}$. No mesmo estudo, quando comparadas amostras de pele e de nervos do mesmo paciente, sabidamente hansênico, a concordância foi de 48\%. Numa classificação histopatológica retrospectiva, Barbosa Jr et al. (1998) ${ }^{11}$ verificaram que em 49,3\% das 1108 biópsias de casos com suspeita clínica de hanseníase os achados histopatológicos eram indistinguíveis de outras dermatites crônicas. Em parte dos 50,7\% onde o diagnóstico histopatológico pôde ser realizado, houve dificuldade na classificação segundo Ridley \& Jopling. Já Alves et al. (2004) encontraram na análise de 1385 laudos de biópsias de pele $32 \%$ de discordância entre a hipótese diagnóstica e o diagnóstico anátomo-patológico, sendo a Hanseníase um dos principais diagnósticos discordantes ${ }^{16}$.

No Serviço de Anatomia Patológica do Hospital de Base de São José do Rio Preto - SP é feito o diagnóstico histopatológico de Hanseníase baseando-se principalmente na classificação de Madrid. $\mathrm{O}$ presente estudo tem como objetivo realizar exame histopatológico retrospectivo de casos de Hanseníase diagnosticados entre 2002 e 2004 e classificá-los segundo os critérios de Ridley \& Jopling, analisando também a sua correlação anátomo-clínica.

\section{2- MATERIAL E MÉTODO}

Foram revisados os resumos de biópsia arquivados no Serviço de Anatomia Patológica do Hospital de Base de São José do Rio Preto dos anos 2002 a 2004, e levantados os laudos completos com os pedidos de biópsia daqueles com diagnóstico definitivo ou compatível com Hanseníase, nesse último quando não foram encontrados bacilos. Dos 82 casos obtidos retiramos dados dos pedidos de biópsia e do laudo histopatológico. Destes, retrocedendo a partir do ano 2004, selecionamos 50 casos, excluindo aqueles com diagnóstico de formas reacionais da Hanseníase ou que não apresentavam material suficiente para revisão histopatológica.

Para classificar os 50 casos segundo os critérios de Ridley \& Jopling (1966) ${ }^{9,10}$, foram analisadas as lâminas já coradas pelos métodos de Hematoxilina e Eosina (H\&E) e Ziehl-Neelsen, utilizando como parâmetros: atrofia da epiderme, zona subepidérmica livre de infiltrado inflamatório (zona de Grenz), distribuição do infiltrado inflamatório, presença de células epitelióides e células gigantes, linfócitos, presença e tipo de granuloma, envolvimento neural e presença e quantidade de bacilos álcool-ácido resistentes (BAAR). Uma dermatopatologista e dois acadêmicos do curso de Medicina, previamente treinados, foram os respon- 
sáveis pela análise, e os três revisaram as 50 lâminas. Os resultados histopatológicos foram classificados entre as formas I, TT, DT, DL, LLs e LLp ${ }^{9,10}$, sem referência ao aspecto clínico, muitas vezes descrito incompletamente. As formas I, TT e DT são paucibacilares, enquanto DL, LLs e LLp são multibacilares ${ }^{2,9}$.

Os dados foram digitados em planilha e analisados no programa Epi Info 2002®, realizando nos cruzamentos o teste do Qui-quadrado com valor alfa de $5 \%$.

\section{3- RESULTADOS}

Os resultados obtidos a partir dos pedidos de biópsia e laudos histopatológicos estão nas Tabelas I e II. A mediana das idades foi 52, com mínimo de 25 e máximo de 85 , com $62 \%$ dos pacientes entre 41 e 60 anos de idade, e os homens predominaram com $60 \%$ dos casos (Tabela I). A biópsia ocorreu nos membros e tronco em $86 \%$ dos casos. Em 25 casos (50\%) foi diagnosticado Hanseníase no pólo lepromatoso, 15 (30\%) no pólo tuberculóide, nove indeterminadas e apenas uma dimorfa (Tabela I).

Quanto à descrição clínica (Tabela II), 19 (38\%) pacientes possuíam lesões em mais de uma região do

Tabela I. Resultados de 50 laudos de biópsias de Hanseníase, São José do Rio Preto, SP, 2006.

\begin{tabular}{llrr}
\hline & & $\mathrm{N}$ & $\%$ \\
\hline Idade & & 7 & 14 \\
& 25 a 40 & 31 & 62 \\
Mediana: 52 & 41 a 60 & 12 & 24 \\
& 61 a 85 & 30 & 60 \\
Sexo & masculino & 20 & 40
\end{tabular}

Diagnóstico de Hanseníase*

$\begin{array}{lrr}\text { indeterminada** } & 9 & 18 \\ \text { tuberculóide** } & 15 & 30 \\ \text { dimorfa } & 1 & 2 \\ \text { lepromatosa } & 25 & 50\end{array}$

Local da biópsia

$\begin{array}{lrr}\text { membro superior } & 15 & 30 \\ \text { tronco } & 14 & 28 \\ \text { membro inferior } & 14 & 28 \\ \text { outros locais } & 4 & 8 \\ \text { não especificado } & 3 & 6\end{array}$

* Segundo a classificação de Madrid (1953).

** ou compatível com a hipótese diagnóstica clínica. corpo, e 27 (54\%) tinham lesões localizadas em apenas uma região. Em 18 pacientes (36\%) fez-se a hipótese diagnóstica clínica (HDC) de Hanseníase, contudo sem especificar forma, enquanto em dois (4\%) foram aventadas outras hipóteses que não Hanseníase. De 30 HDCs especificadas de Hanseníase, as quatro formas foram citadas número semelhante de vezes (entre sete e treze), contudo geralmente se pensou em mais de uma hipótese por paciente.

A classificação das formas histopatológicas segundo os critérios de Ridley \& Jopling se encontra na Tabela III, juntamente com a percentagem de casos em que foram encontrados bacilos álcool-ácido resistentes (BAAR), independente da quantidade. A forma mais freqüente foi a polar lepromatosa (LLp), com 16 casos $(32 \%)$, seguida pela polar tuberculóide (TT) e indeterminada (I), com 10 e 9 casos respectivamente. Todas as lâminas das formas dimorfa lepromatosa (DL) e subpolar e polar lepromatosas (LLs e LLp) continham bacilos, enquanto em uma dimorfa tuberculóide (DT) não foram encontrados bacilos e três indeterminadas os continham em pequena quantidade. Na Tabela III também se encontra o número de casos multibacilares e paucibacilares.

Tabela II. Aspectos clínicos referidos nos pedidos de biópsia, SJRP, SP, 2006. $\mathrm{N}=50$.

\begin{tabular}{lrr}
\hline & $\mathrm{N}$ & \% \\
\hline Localização das lesões & & \\
membro superior & 10 & 20 \\
membro inferior & 10 & 20 \\
tronco ou cabeça & 7 & 14 \\
lesões disseminadas* & 19 & 38 \\
não especificada & 4 & 8
\end{tabular}

\section{Hipótese Diagnóstica Clínica}

de Hanseníase

não especificada $\quad 18 \quad 36$

especificada $\quad 30 \quad 60$

outra hipótese** $\quad 2 \quad 4$

Forma especificada na

Hipótese Clínica***

indeterminada $\quad 12$

tuberculóide $\quad 13$

dimorfa 10

lepromatosa 7

reacional 3

* Em mais de uma das localizações anteriores

** Hipótese clínica não compatível com a histopatologia

*** Cada pedido pode conter mais de uma hipótese clínica 


\begin{tabular}{|c|c|c|c|c|}
\hline & \multicolumn{4}{|c|}{ Presença d } \\
\hline & \multicolumn{2}{|c|}{ Forma } & \multicolumn{2}{|c|}{ Bacilos } \\
\hline & $\mathrm{N}$ & $\%$ & $\mathrm{~N}$ & \\
\hline Indeterminada & 9 & 18 & 3 & 33 , \\
\hline Tuberculóide & 10 & 20 & 0 & \\
\hline Dimorfa Tuberculóide & 5 & 10 & 4 & \\
\hline Dimorfa Lepromatosa & 5 & 10 & 5 & \\
\hline Subpolar Lepromatosa & 5 & 10 & 5 & \\
\hline Lepromatosa & 16 & 32 & 16 & \\
\hline Multibacilar* & 26 & 52 & & \\
\hline Paucibacilar** & 24 & 48 & & \\
\hline
\end{tabular}

${ }^{*}$ Formas I, TT e DT.

** Formas DL, LLs e LLp

A forma multibacilar predominou no sexo masculino, com prevalência de $66,7 \%(\mathrm{~N}=20 / 30)$, enquanto no sexo feminino $30 \%(\mathrm{~N}=6 / 20)$ apresentaram essa forma $(\mathrm{p}<0,05)$.

Quando investigado o diagnóstico histopatológico anterior, que utilizou a classificação de Madrid, e comparado com a nova classificação, pôde ser observada redistribuição de alguns casos previamente diagnosticados como Hanseníase tuberculóide e lepromatosa (Tabela IV). Das 15 tuberculóides, cinco (33\%) ficaram como DT, e das 25 lepromatosas, quatro (16\%) foram classificadas como DL, cinco $(20 \%)$ como LLs e 16 (64\%) como LLp. A única dimorfa anterior foi classificada como DL.

$\mathrm{Na}$ Tabela $\mathrm{V}$ se encontra a classificação com base na HDC relatada nos pedidos de biópsia, dentre os 30 com forma clínica suspeita especificada (Tabela II). Assim, dos doze casos em que houve suspeita de Hanseníase indeterminada, apenas cinco corresponderam à forma indeterminada no exame histopatológico, enquanto quatro foram classificados na forma tuberculóide, dois na dimorfa tuberculóide e um na subpolar lepromatosa. Por outro lado, dentre oito casos diagnosticados de hanseníase indeterminada, em cinco se suspeitou desse diagnóstico. Essa relação complexa se deve à possibilidade de ter mais de uma forma de Hanseníase na HDC.

Ainda na Tabela V, nota-se que de sete casos TT, em cinco se fez a HDC de Hanseníase tuberculóide; de cinco dimorfas (DT + DL) apenas em um se suspeitou desse diagnóstico; e das dez lepromatosas

Tabela IV. Comparação entre o diagnóstico histopatológico anterior e a nova classificação segundo Ridley \& Jopling, SJRP, SP. N=50.

\begin{tabular}{lrcrrrrr}
\hline Critérios & & \multicolumn{5}{c}{.Ridley \& Jopling [N (\%)] } \\
Madrid & $\mathrm{N}$ & $\mathrm{I}$ & $\mathrm{TT}$ & $\mathrm{DT}$ & $\mathrm{DL}$ & LLs & LLp \\
\hline Indeterminada & 9 & $9(100)$ & & & & & \\
Tuberculóide & 15 & & $10(67)$ & $5(33)$ & & & \\
Dimorfa & 1 & & & & $1(100)$ & & \\
Lepromatosa & 25 & & & & $4(16)$ & $5(20)$ & $16(64)$ \\
\hline
\end{tabular}

Tabela V. Formas suspeitas na HDC* de Hanseníase e classificação histopatológica, SJRP, SP. N=30.

\begin{tabular}{|c|c|c|c|c|c|c|c|}
\hline \multirow[b]{2}{*}{$\mathrm{HDC}$} & \multicolumn{7}{|c|}{ Classificação Ridley \& Jopling } \\
\hline & $\mathrm{N}^{* *}$ & I & TT & DT & DL & LLs & LLp \\
\hline Indeterminada & 12 & 5 & 4 & 2 & & 1 & \\
\hline Tuberculóide & 13 & 3 & 5 & 2 & 1 & 1 & 1 \\
\hline Dimorfa & 10 & 3 & 1 & & 1 & & 5 \\
\hline Lepromatosa & 7 & 2 & & & 1 & 2 & 2 \\
\hline Reacional & 3 & & 1 & & & & 2 \\
\hline Total*** & 30 & 8 & 7 & 3 & 2 & 3 & 7 \\
\hline
\end{tabular}

* Hipótese diagnóstica clínica.

${ }^{* *}$ Poderia haver mais de uma suspeita por caso.

*** Considera apenas os casos com HDC de Hanseníase com forma especificada. 
(LLs + LLp) as HDCs foram principalmente cinco dimorfas, quatro lepromatosas e cinco de outras formas. Em suma, a suspeita clínica parece ser mais confiável para as formas I e TT, apesar de em todas as formas ter sido diversificada a HDC quando comparada com o diagnóstico histopatológico. Foram aventadas diversas outras hipóteses para o quadro clínico dos pacientes, por exemplo (em ordem decrescente de freqüência): eritema polimorfo, eritema pigmentado fixo, tinhas, pitiríase, urticária, granuloma anular, sífilis secundária, leishmaniose, micoses sistêmicas, dentre outras.

\section{4- DISCUSSÃo}

A pesquisa através dos resumos de biópsias, que continham apenas o início do laudo histopatológico, dificultou o encontro dos casos de Hanseníase, e possivelmente não foram encontrados todos os casos diagnosticados no serviço entre 2002 e 2004, contudo sem prejuízo para o objetivo do estudo. Os pedidos de biópsia, com dados clínicos e epidemiológicos, raramente apresentaram resultados do teste da lepromina ou da baciloscopia, e em 36\% não houve HDC de forma específica de Hanseníase (Tabela II), dificultando uma classificação mais exata dos casos, que dependem desses dados além da histopatologia 2,9,10,12,18,19. Barbosa Jr et al. (1998) e Vargas-Ocampo (2004) também relataram "lacunas" de informação nas requisições para biópsia, em estudos na Bahia com 1108 biópsias e no México com 6000 formulários de biópsia, respectivamente ${ }^{11,20}$.

A maior prevalência de Hanseníase encontrada no sexo masculino (Tabela III) é relatada na literatura $^{2,5,11,13}$, assim como a maior prevalência de casos multibacilares nesse gênero, que foi observada por Vargas-Ocampo no México ${ }^{20}$, e é descrita no Brasil ${ }^{2}$. Tal distribuição pode estar relacionada a questões sócio-culturais, como a menor procura de auxílio médico pelo homem, e a uma suposta maior suscetibilidade às formas multibacilares ${ }^{2}$. A elevada porcentagem de pacientes com forma lepromatosa polar (32\%, Tabela III) indica o diagnóstico tardio da doença, dado relevante por se tratar de forma infectante ${ }^{2}$, e com maiores índices de incapacidade física ${ }^{13}$.

Apesar de no Brasil haver maior proporção de casos multibacilares ${ }^{1,2,3}$, no presente estudo o número foi semelhante ao de paucibacilares (52 e $48 \%$ respectivamente, Tabelas I e III), enquanto no Estado da Bahia, Barbosa Jr et al. (1998) encontraram 86,2\% de paucibacilares ${ }^{11}$, e Aquino et al. (2003) relataram $50,7 \%$ de multibacilares na Amazônia do Maranhão ${ }^{13}$. A epidemiologia diversa nas regiões do Brasil poderia ser devido às características sócio-culturais, como relatado para os homens anteriormente, ou estar ligada à predisposição genética prevalente em cada região, pois é observada em estudos recentes a distribuição das formas de Hanseníase segundo caracteres genéticos individuais, que proporcionam a resposta anérgica ou alérgica ao $M$. leprae 1,2,7,21 $_{\text {. }}$

$\mathrm{Na}$ Tabela III se encontra a classificação dos casos de Hanseníase nas formas descritas por Ridley \& Jopling e a porcentagem de lâminas com bacilos. Barbosa Jr et al. (1998) encontraram resultados semelhantes, com bacilos em $43,1 \%$ das indeterminadas, em nenhuma das TT, em $66,2 \%$ das DT, e em todas as DD, DL e LL ${ }^{11}$. Na forma TT há principalmente granulomas tuberculóides em toda a profundidade da derme, com células epitelióides bem diferenciadas, células gigantes tipo Langerhans e tipo corpo estranho, e linfócitos em torno do granuloma ou entre as células epitelióides. O diagnóstico vai depender da detecção de granulomas endoneurais, visto que na maioria das vezes a baciloscopia é negativa. Em certas lesões suspeitas de TT não é possível detectar bacilos ou estruturas neurais. Essa apresentação pode estar indicando a total destruição destes últimos. Porém, este não é um critério confiável para diagnóstico, pois reações granulomatosas extensas podem ocultar os nervos ${ }^{22}$. Quando a presença dessas características estiver associada a informações clínicas confiáveis, principalmente quanto a alterações da sensibilidade nas lesões, o diagnóstico é compatível com Hanseníase tuberculóide ${ }^{9,10}$.

A forma DT apresenta quadro histopatológico semelhante ao TT, porém a baciloscopia pode ser positiva e os nervos são mais preservados. Assim, a baciloscopia é importante na diferenciação entre DT e TT. Enquanto a biópsia demonstrar padrão tuberculóide, independente de baciloscopias elevadas, o diagnóstico continua sendo $\mathrm{DT}^{22}$. A resistência parcial dos DT ou do grupo dimorfo como um todo, propicia a proliferação de uma parcela dos bacilos, com conseqüente aumento progressivo da quantidade destes nas lesões ${ }^{9,10,22}$. No subgrupo DL predominam granulomas extensos, constituídos por macrófagos modificados não epitelióides e há um denso infiltrado linfocitário. Os nervos mostram, freqüentemente, delaminação do perineuro por linfócitos. Com a progressão para a forma LLp, passando pela LLs, ocorre desorganização 
do granuloma, constituído por macrófagos multivacuolados, diminuição marcante do número de linfócitos, envolvimento neural mínimo e alta densidade de bacilos, que podem se apresentar íntegros ou granulosos ${ }^{23}$.

Na Tabela IV está a comparação da classificação segundo Ridley \& Jopling com o diagnóstico anterior, em que pode ser notada igual proporção de formas multibacilares e paucibacilares. Esses resultados demonstram que a classificação histopatológica da Hanseníase pode ser difícil mesmo quando se usam critérios semelhantes ${ }^{9,11,20}$, dependendo também da experiência dos examinadores, apesar das conclusões semelhantes alcançadas (Tabela IV). Como já observado, o diagnóstico e a classificação corretos permitem tratamento adequado e previsão do prognóstico e evolução do paciente, daí a importância de sua padronização e aplicação ${ }^{1,2,9,14}$. Apesar desse conhecimento e de décadas de estudo, não parece ter sido estabelecido um consenso na classificação de Ridley \& Jopling, pois alguns autores não utilizam a forma $\mathrm{DD}^{10,19}$, enquanto outros utilizam essa e não a $L_{L s}{ }^{3,11,13}$, e alguns incluem estas e outras formas descritas ${ }^{2,24}$. Devido à instabilidade das formas não polares e à dificuldade da análise histopatológica, uma padronização de quais formas devem ser consideradas é difícil, e pesquisas baseadas na sua resposta terapêutica e surgimento de formas reacionais ainda são importantes ${ }^{15,21}$. Indivíduos apresentando as formas intermediárias e não tratados podem mudar suas características clínicas e histopatológicas, e exibir um quadro clínico e histopatológico inicial diferente da sua verdadeira classificação no espectro da Hanseníase. Alguns, mais próximos do pólo virchowiano, podem manifestar características idênticas às da forma lepromatosa polar 23. Mesmo assim, e apesar do progresso nas pesquisas de métodos diagnósticos na hanseníase, a histopatologia mantém-se como o teste "gold standart" para o seu diagnóstico ${ }^{21}$.

Ao considerar a HDC descrita nos pedidos de biópsia (Tabela V), foi freqüente a discordância entre estas e o diagnóstico histopatológico. Entretanto, não foi possível quantificar esta discordância devido à existência de múltiplas HDCs na maioria dos pedidos. $\mathrm{O}$ diagnóstico diferencial de Hanseníase inclui muitas doenças, e é comum a não correlação entre a clínica e a histopatologia das lesões suspeitas de Hanseníase $1,11,16,17,19,20,25,26$. Na avaliação morfológica dos granulomas, especialmente no sentido do pólo tuberculóide, não é possível distinguir a Hanseníase de outras dermatoses granulomatosas, como a Paracocci- dioidomicose sarcoídica, a Sífilis secundária tardia e a Sarcoidose ${ }^{22}$. O diagnóstico depende de outras informações, como a detecção de bacilos álcool-ácido resistentes, do quadro clínico, número e distribuição das lesões, dentre outras. Em 1966, Ridley \& Jopling analisaram 82 pacientes e houve correlação clínicahistológica completa em 56 casos, enquanto 21 apresentaram pouca discordância e 5 séria discordância ${ }^{9}$. Considerando ainda outra forma de classificação, baseada apenas na clínica, Rao et al. (2006) avaliaram 77 pacientes com formas clinicamente paucibacilares (de uma a cinco lesões) e encontraram 63\% de correlação clínico-histopatológica para a forma dimorfa tuberculóide, e quatro casos de forma dimorfa lepromatosa ${ }^{19}$. Em 2005, Reddy et al. ${ }^{26}$ avaliaram 27 pacientes não tratados, com lesão única e sem comprometimento neural clínico, encontrando correlação clínicopatológica da pele de $51,8 \%$, entre pele e nervo de $26 \%$, e em $55,5 \%$ os nervos estavam acometidos. $\mathrm{Na}$ análise de biópsias de pele aparentemente normal de 50 pacientes com hanseníase, El-Darouti et al. $(2006)^{27}$ encontraram alterações características da doença em $52 \%$ dos casos. Portanto, a análise de diferentes métodos classificatórios da hanseníase reforça a importância da associação da clínica com a histopatologia, além de outros dados, para a classificação dessa doença, que continua desafiando os pesquisadores ${ }^{21}$.

\section{5- CONCLUSÕES}

O diagnóstico de hanseníase e a classificação correta de suas diferentes formas dependem de atenção aos critérios histológicos, clínicos, imunológicos (teste de Mitsuda) e da correlação entre os mesmos visando um tratamento eficaz do paciente.

Há dificuldade para se utilizar a classificação de Ridley \& Jopling no diagnóstico histopatológico de hanseníase devido à falta de dados clínicos e imunológicos, que são necessários para a conclusão diagnóstica. Quando comparada com a classificação de Madrid, houve diferenças em algumas formas, contudo com poucas implicações no tratamento, por não se alterar a forma quanto à quantidade de bacilos.

É necessário treinamento de médicos clínicos e patologistas visando a conscientização da importância da correlação dos dados clínicos, histopatológicos e imunológicos para conclusão diagnóstica de hanseníase bem como para especificação de suas diferentes formas, em vista da freqüente discordância anátomo-clínica. 
Pavani RAB, Tonolli ER, D'Avila SCGP. Histopathological classification and clinical correlation of 50 leprosy cases from a Teaching Hospital, São José do Rio Preto, São Paulo state, Brazil. Medicina (Ribeirão Preto) 2008; 41 (2): 188-95.

SUMMARY: The classification of leprosy most used in clinical practice is that adapted by the Madrid Congress (1953), which contains the indeterminate, tuberculoid, borderline and lepromatous forms. Ridley \& Jopling have proposed in 1996 a classification based on the immunological spectrum of the disease. Design of the study: Prevalence survey. Objective: To classify leprosy biopsies according to Ridley and Jopling classification and perform the clinicopathological correlation. Methodology: Fifty biopsy solicitations were reviewed and their slides stained by H\&E and Ziehl-Neelsen methods were analyzed. Results: There were found: 9 indeterminate, 10 tuberculoid, 5 borderline tuberculoid, 5 borderline lepromatous, 5 subpolar lepromatous and 16 polar lepromatous forms. From 50 patients, 30 were male, and the multibacilar forms predominated in this gender. Leprosy diagnostic hypothesis was not formulated in 2 subjects and in 30 cases one or more forms were specified, with frequent disagreement between histopathological diagnostic and clinical hypothesis. Conclusions: The classification of leprosy requires attention to the histopathological criteria and correlation with patients' clinical and immunological information.

Descriptors: Leprosy. Pathology. Classification. Histopathological Diagnosis. Clinical.

\section{REFERÊNCIAS}

1 - Araújo MG. Hanseníase no Brasil. Rev Soc Bras Med Trop 2003; 36(3): 373-82.

2 - Margarido LC, Rivitti EA. Hanseníase. In: Focaccia R, editor. Veronesi: Tratado de Infectologia. $3^{\text {a }}$ edição. São Paulo: Editora Atheneu; 2005. Cap 54; p. 939-72.

3 - Opromolla DV, Nóbrega RC, Gonçalves NNS, Padovani SHP, Padovani CR, Gonçalves A. Estimativa da prevalência da hanseníase pela investigação em demanda inespecífica de agências de saúde. Rev Saúde Pública 1990; 24: 178-85.

4 - Helene LMF, Salum MJL. A reprodução social da hanseníase: um estudo do perfil de doentes com hanseníase no Município de São Paulo. Cad Saúde Pública 2002; 18(1):101-13.

5 - Neves RG. Hanseníase. In: Cucé LC, Neto CF, editores. Manual de dermatologia. $2^{a}$ edição. São Paulo: Editora Atheneu; 2001. Cap 10.2; p. 132-58.

6 - Abulafia J, Vignale RA. Leprosy: pathogenesis updated. Int J Dermatology 1999; 38: 321-34.

7 - Goulart IMB, Penna GO, Cunha G. Imunopatologia da hanseníase: a complexidade dos mecanismos da resposta imune do hospedeiro ao Mycobacterium leprae. Rev Soc Bras Med Trop 2002; 35(4): 365-75.

8 - [Congress, Madrid]. Classification. Technical Resolutions, VIth International Congress Leprol. Madrid, 1953. Int J Lepr 1953; 21: $504-16$

9 - Ridley DS, Jopling WH. Classification of Leprosy According to Immunity - A five-group system. Int J Leprosy 1966; 34(3): 255-73.
10 - Fields JP, Meyers WM. Mycobacterial Infections: Leprosy. In: Farmer ER, Hood AF, editors. Pathology of the skin. 2nd edition. New York: McGraw-Hill; 2000. Chap 29; p. 557-70.

11 - Barbosa Júnior AA, Jambeiro J, Cirqueira JSO, Silva TC. Classificação histopatológica retrospectiva de 1108 biópsias de pele de pacientes com suspeita clínica de hanseníase provenientes do Estado da Bahia, nordeste do Brasil. Rev Soc Bras Med Trop 1998; 31(6): 533-7.

12 - Murthy PK. Clinical manifestations, diagnosis and classification of leprosy. J Indian Med Assoc 2004; 102(12): 678-9.

13 - Aquino DMC, Caldas AJM, Silva AAM, Costa JML. Perfil dos pacientes com hanseníase em área hiperendêmica da Amazônia do Maranhão, Brasil. Rev Soc Bras Med Trop 2003; 36(1): 57-64.

14 - Kociecka W. Contemporary problems in the epidemiology and clinical pathology of leprosy. Wiad Parazytol 1995; 41(4): 373-90.

15 - Nery JAC, Vieira LMM, Matos HJ, Gallo MEN, Sarno EN. Reactional states in multibacillary Hansen disease patients during multidrug therapy. Rev Inst Med Trop S Paulo 1998;40(6):363-70.

16 - Alves JR, Hida M, Nai GA. Diagnóstico clínico e anátomopatológico: Discordâncias. Rev Assoc Med Bras 2004; 50 (2): 178-81.

17 - Khan A, Koranne RV, Bajaj P, Ravi B. Clinico-histopathological correlation of skin and nerve in leprosy. J Dermatol 2004; 31(8): 632-6.

18 - Ramos-E-Silva M, Oliveira ML, Munhoz-da-Fontoura GH. Leprosy: uncommon presentations. Clin Dermatol 2005; 23(5): 509-14. 
19 - Rao PN, Pratap DVS, Ramana Reddy AV, Sujai S. Evaluation of leprosy patients with 1 to 5 skin lesions with relevance to their grouping into paucibacillary or multibacillary disease. Indian J Dermatol Venereol Leprol 2006; 72: 207-10.

20 - Vargas-Ocampo F. Análisis of 6000 skin biopsies of the national leprosy control program in Mexico. Int J Lepr Other Mycobact Dis 2004; 72(4): 427-36.

21 - Scollard DM, Adams LB, Gillis TP, Krahenbuhl JL, Truman RW, Williams DL. The continuing challenges of leprosy. Clinical Microbiology Reviews 2006; 19 (2): 338-81.

22 - Fleury RN. Critérios para o emprego de classificação de Ridley e Jopling na rotina dos laboratórios de anatomia patológica: primeira parte. Hansen Int 2005; 30(2): 201-5.

23 - Fleury RN. Critérios para o emprego de classificação de Ridley e Jopling na rotina dos laboratórios de anatomia patológica: segunda parte. Hansen Int 2006; 31(1) [online] [citado 30 de Mar de 2008]. Disponível em http://www.ilsl.br/ revista/index.php/hi/article/view/22/9.
24 - Margarido LC. Hanseníase. In: Sampaio SAP, Rivitti EA, editores. Dermatologia. $3^{a}$ edição. São Paulo: Editora Artes Médicas; 2007. Cap 41; p. 625-51.

25 - Singhi MK, Kachhawa D, Ghiya BC. A retrospective study of clinico-histopathological correlation in leprosy. Indian J Pathol Microbiol 2003; 46(1): 47-8.

26 - Reddy RR, Singh G, Sacchidanand S, Okade R, Shivakumar $V$, Uday A et al. A comparative evaluation of skin and nerve histopathology in single skin lesion leprosy. Indian J Dermatol Venereol Leprol 2005; 71(6): 401-5.

27 - El-Darout MA, Hussein S, Marzouk SA, Nabil N, Hunter NS, Mahgoub $D$ et al. Histopathological study of apparently normal skin of patients with leprosy. Int J Dermatol 2006; 45(3): 292.

Recebido para publicação em 23/07/2007.

Aprovado para publicação em 12/05/2008. 